OPEN ACCESS

Edited by:

Matteo Becatti,

University of Florence, Italy

Reviewed by:

Manoj Kumar Kashyap,

Amity University, Gurgaon, India

Dragos Cretoiu,

Carol Davila University of Medicine and Pharmacy, Romania

*Correspondence:

Hongjun Zhu

28254282@qq.com

†These authors have contributed equally to this work

Specialty section:

This article was submitted to

Molecular Diagnostics

and Therapeutics,

a section of the journal

Frontiers in Molecular Biosciences

Received: 22 February 2021

Accepted: 19 April 2021

Published: 19 May 2021

Citation:

Liu J, Xu M, Wu Z, Yang Y, Yuan S, Liang J and Zhu H (2021) Low Expression of RILPL2 Predicts Poor Prognosis and Correlates With Immune Infiltration in Endometrial

Carcinoma.

Front. Mol. Biosci. 8:670893. doi: 10.3389/fmolb.2021.670893

\section{Low Expression of RILPL2 Predicts Poor Prognosis and Correlates With Immune Infiltration in Endometrial Carcinoma}

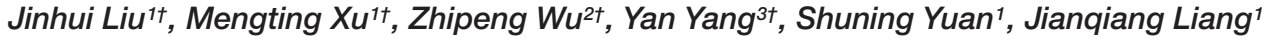 \\ and Hongjun $\mathrm{Zhu}^{4 *}$
}

\begin{abstract}
${ }^{1}$ Department of Gynecology, The First Affiliated Hospital of Nanjing Medical University, Nanjing, China, ${ }^{2}$ Department of Urology, The Affiliated Sir Run Run Hospital of Nanjing Medical University, Nanjing, China, ${ }^{3}$ Department of Diagnosis, The first Clinical Medical College of Nanjing Medical University, Nanjing, China, ${ }^{4}$ Department of Oncology, Nantong Third People's Hospital Affiliated to Nantong University, Nantong, China
\end{abstract}

Increasing numbers of biomarkers have been identified in various cancers. However, biomarkers associated with endometrial carcinoma (EC) remain largely to be explored. In the current research, we downloaded the RNA-seq data and corresponding clinicopathological features from the Cancer Genome Atlas (TCGA) database. We conducted an expression analysis, which resulted in RILPL2 as a novel diagnostic biomarker in EC. The dysregulation of RILPL2 in EC was also validated in multiple datasets. The correlations between clinical features and RILPL2 expression were assessed by logistic regression analysis. Then, Kaplan-Meier analysis, univariate and multivariate Cox regression analysis were performed to estimate prognostic values of RILPL2 in the TCGA cohort, which revealed that increased level of RILPL2 was remarkably associated with better prognosis and could act as an independent prognostic biomarker in patients with EC. Moreover, correlation analysis of RILPL2 and tumor-infiltrating immune cells (TIICS) indicated that RILPL2 might play a critical role in regulating immune cell infiltration in $\mathrm{EC}$ and is related to immune response. Besides, high methylation level was a significant cause of low RILPL2 expression in EC. Subsequently, weighted gene co-expression network analysis (WGCNA) and enrichment analysis were conducted to explore the RILPL2-involved underlying oncogenic mechanisms, and the results indicated that RILPL2 mainly regulated cell cycle. In conclusion, our findings provided evidence that downregulation of RILPL2 in EC is an indicator of adverse prognosis and RILPL2 may act as a promising target for the therapeutics of EC.

Keywords: RILPL2, endometrial carcinoma, prognosis, immune infiltration, biomaker

\footnotetext{
Abbreviations: CDR, clinical data resource; DCs, dendritic cells; DEGs, differentially expressed genes; DFS, disease-free survival; DSS, disease-specific survival; EC, endometrial carcinoma; GEO, gene expression omnibus; GO, gene ontology; GS, gene significance; HCV, Hepatitis C virus; TCGA, the Cancer Genome Atlas; HPA, human protein atlas; KEGG, kyoto encyclopedia of genes and genomes; ME, module eigengene; MM, module membership; MS, module significance; OS, overall survival; PPI, protein-protein interaction; qRT-PCR, real-time quantitative RT-PCR; RILP, Rab-interacting lysosomal protein; RILPL2, Rab interacting lysosomal protein like 2; TIICs, tumor-infiltrating immune cells; TOM, top logical overlap matrix; WGCNA, weighted gene co-expression network analysis.
} 


\section{INTRODUCTION}

Endometrial carcinoma (EC) is the fourth most common malignant tumors among females in the world behind breast cancer, lung cancer, and colorectal cancer (Sorosky, 2012), with increasing incidence and morbidity over the past years (Siegel et al., 2019). To differentiate the prognosis and its clinical course, The experts divided EC into type I and type II. Type I EC is characterized by estrogen-dependent pathogenesis, low-grade lesions and better clinical outcome, while type II EC is regarded as non-estrogen-dependent, poorly differentiated and has poorer prognosis (Bokhman, 1983). Although most ECs are generally early-stage and low-grade with favorable prognosis, the highgrade group accounts for a disproportionate number of EC deaths (Creasman et al., 2004). Despite EC still occurs more commonly in senior women, it also is being diagnosed in younger women (Moore and Brewer, 2017). Therefore, it is urgent to explore potential indicators that are directly associated with diagnosis in the early stage, therapy, prognosis after treatment and detection of asymptomatic recurrence in endometrial carcinoma.

Rab interacting lysosomal protein like 2 (RILPL2) is a proteincoding gene, encoding a protein that contains a Rab-interacting lysosomal protein-like domain similar to that of Rab-interacting lysosomal protein (RILP). RILP is a Rab7/Rab34 effector which interacts with dynein-dynactin, in order to directly regulate the movement of late endosomes and lysosomes along with microtubules (Johansson et al., 2007). Unlike RILP, RILPL2 does not consist of a Rab7 junction region, which prevents its ectopic expression from the lysosomal compartment (Wang et al., 2004). Although RILPL2 does not affect lysosomal trafficking, it may still act as a functional Rab effector due to its interaction with activated Rab34 and Rab36 (Fukuda et al., 2008; Matsui et al., 2012). The analysis of data obtained from online databases suggested that RILPL2 expressed lower in breast cancer tissues than that in adjacent tissues, and that low RILPL2 expression was prominently positively associated with TNM stage and differentiated grade (Chen et al., 2019). Furthermore, previous studies indicated that RILPL2 functioned in viral replication and could be employed as a potential target for the therapy of Hepatitis C virus (HCV) (Dolan et al., 2013). However, the correlation between RILPL2 and the clinical features of EC has not been studied.

In this research, we first analyze the expression level of RILPL2 and summarize its prognostic roles, biological features, and correlation with tumor immune infiltration in EC patients, mainly according to the public data obtained from the Cancer Genome Atlas (TCGA) database. Summarily, our study suggests that RILPL2 may be a novel predictive indicator for diagnosis, prognosis and promising therapeutic target for EC.

\section{MATERIALS AND METHODS}

\section{Collection of the TCGA and the GEO Data}

Transcriptome RNA-seq data of 575 samples (containing 23 normal samples and 552 tumor samples) and the corresponding clinical characteristics were downloaded from the TCGA database ${ }^{1}$, which acted as a public repository that includes highthroughput microarray experimental data (Liu et al., 2018). EC sequencing data were generated by the Illumina HiSeq RNA-Seq platform. Then, we conducted pre-processing of clinicopathologic data to eliminate cases with missing or defective information. As a result, the clinical information of 524 patients were reserved for the following analysis.

The GSE17025 dataset was obtained from the Gene Expression Omnibus (GEO) database ${ }^{2}$ (Day et al., 2011), which was used to validate the expression of RILPL2. The GSE17025 dataset contained high-throughput data of 91 EC samples, and 12 atrophic endometrium samples from postmenopausal women.

\section{Collection of EC Specimens}

To validate the aberration of RILPL2 in EC tissues compared to normal tissues, we further collected $15 \mathrm{EC}$ tissues and paired adjacent tissues in the Third People's Hospital of Nantong in 2020. Ethical approval for the study was granted by the Clinical Research Ethics Committee, the Third People's Hospital of Nantong, and the current study was performed in conform to the Declaration of Helsinki.

\section{Total RNA Extraction and Quantitative Real-Time PCR Analysis}

To extract total RNA from EC tissues, TRIzol reagent (Thermo Fisher Scientific) was applied. Next, Agilent Bioanalyzer 2100 (Agilent Technologies) with RNA 6000 Nano kit was used to assess the integrity of extracted total RNA. All procedures of real-time quantitative RT-PCR (qRT-PCR) are described as previously (Liu et al., 2020a). Primer sequences for $\mathrm{qRT}$-PCR were presented as follows: RILPL2: ACGTGTATGACATCTCCTACCTG (forward), ACGCGGACGACTTTGAACTG (reverse); GAPDH: CGCT CTCTGCTCCTCCTGTT (forward), CATGGGTGGAATCAT ATTGG (reverse).

\section{Validation of RILPL2 Protein Level}

Human Protein Atlas (HPA), an integration of multi-omics, puts insight into characterizing all human proteins (Uhlen et al., 2015). In this study, the level of RILPL2 in human endometrium and EC samples were compared after acquiring data from the HPA database. Besides, to further validate the protein level of RILPL2, the CPTAC analysis in UALCAN was applied (Chandrashekar et al., 2017).

\section{Analysis of RILPL2 Expression in EC Samples With Various Clinicopathologic Features}

For the data downloaded from the TCGA database, we utilized "ggplot" package in R language (v. 3.6.2) to analyze the RNAseq data and corresponding clinical features. The relevance between the expression of RILPL2 and various clinicopathologic characteristics was evaluated and emerged by boxplots. The

\footnotetext{
${ }^{1}$ https://portal.gdc.cancer.gov/

${ }^{2}$ www.ncbi.nlm.nih.gov/geo
} 
difference of variables was tested using the Wilcoxon signedrank test or Kruskal-Wallis test. A logistic regression analysis of RILPL2 level and other clinical features was performed via $\mathrm{R}$ language. $P<0.05$ was considered as statistically significant.

\section{Survival Analysis Dependent on RILPL2 Expression}

Definitions and outcomes of overall survival (OS), diseasefree survival (DFS), and disease-specific survival (DSS) of the TCGA cohort were obtained from the TCGA-Clinical Data Resource (CDR) Outcome. Kaplan-Meier curves were exhibited through the "survival" and "survminer" package in $\mathrm{R}, P<0.05$ was considered to be statistically significant. Univariable and multivariable Cox regression analysis were performed orderly to screen out the prognosis-related factors among the available clinical features, together with the RILPL2 expression profile via the "coxph function" in the "survival" package.

\section{Correlation Analysis of RILPL2 and Tumor-Infiltrating Immune Cells}

ESTIMATE, a method which utilizes the gene expression profiles to speculate the infiltrations of stromal and immune cells in tumor tissues (Yoshihara et al., 2013), was applied to assess the level of immune cell content (immune score), the stromal cell infiltration (stromal score), the stromal-immune comprehensive score (ESTIMATE score) and the tumor purity in each EC sample.

The TIMER tool, an online platform ${ }^{3}$ for comprehensively analyzing the molecular features of tumor-infiltrating immune cells (Li et al., 2017), was applied to study and visualize the correlations between the expression of RILPL2 and the proportion of six tumor-infiltrating immune cells (TIICs), including B cells, CD8 + T cells, CD4 + T cells, macrophages, dendritic cells (DCs) and neutrophils.

CIBERSORT $^{4}$, an analytical tool, was applied to precisely assess the infiltration level of TIICs according to the transcriptome data (Newman et al., 2015). According to the infiltration level of 22 immune cell subtypes reported by the CIBERSORT algorithm, the "vioplot" package was applied to visualize the difference of 22 TIICs in EC samples which detected high and low RILPL2 expression.

\section{Methylation and Mutation Analysis}

On the basis of DNA methylation data downloaded from the TCGA database, we assessed the difference of methylation

\footnotetext{
${ }^{3}$ https://cistrome.shinyapps.io/timer/

${ }^{4}$ http://cibersort.stanford.edu/
}
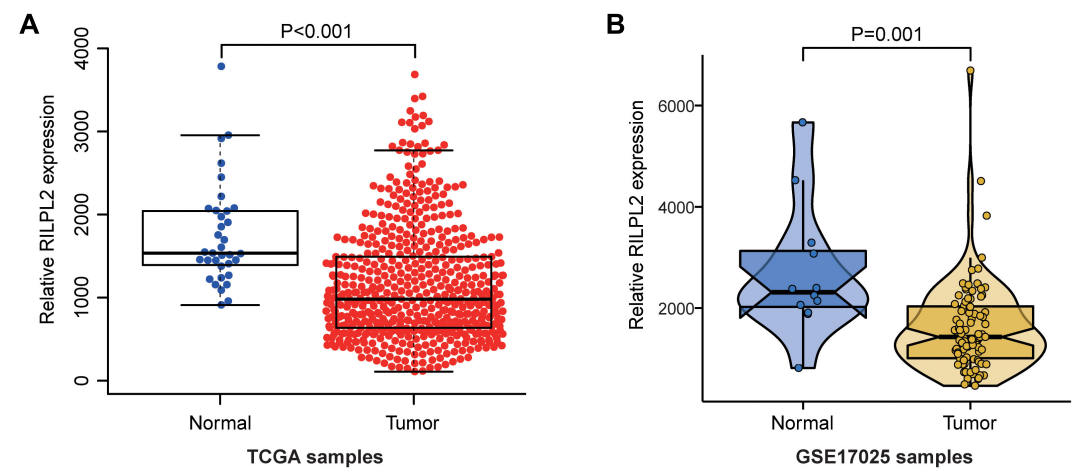

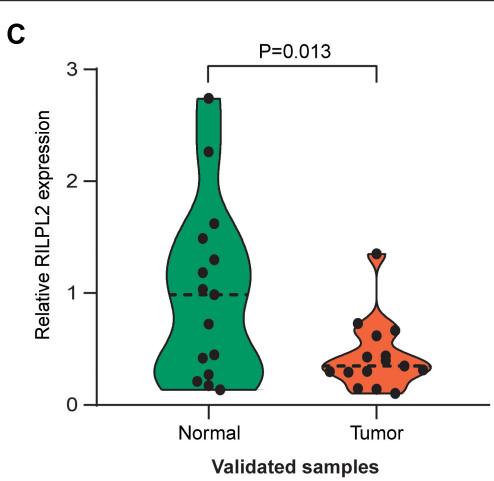

E

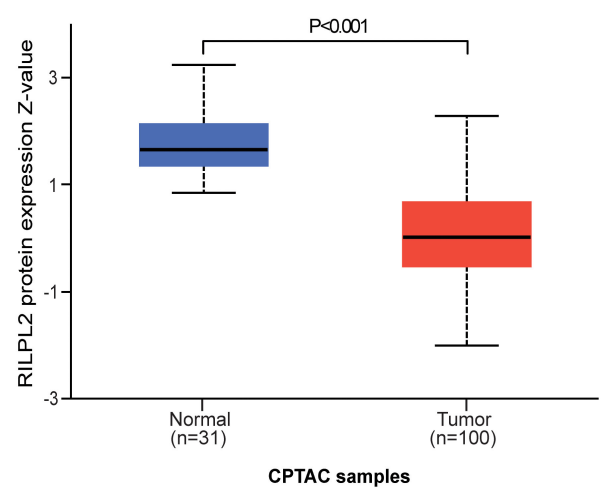

FIGURE 1 | Expression analysis of RILPL2 in EC. (A-C) Differential expression of RILPL2 in tumor and adjacent samples from the TCGA dataset, the GSE17025 dataset, and our recruited cohort. (D) Representative images shows the samples stained with RILPL2 from the HPA database. (E) Differential expression of RILPL2 protein in tumor and adjacent samples from the CPTAC dataset. 
A

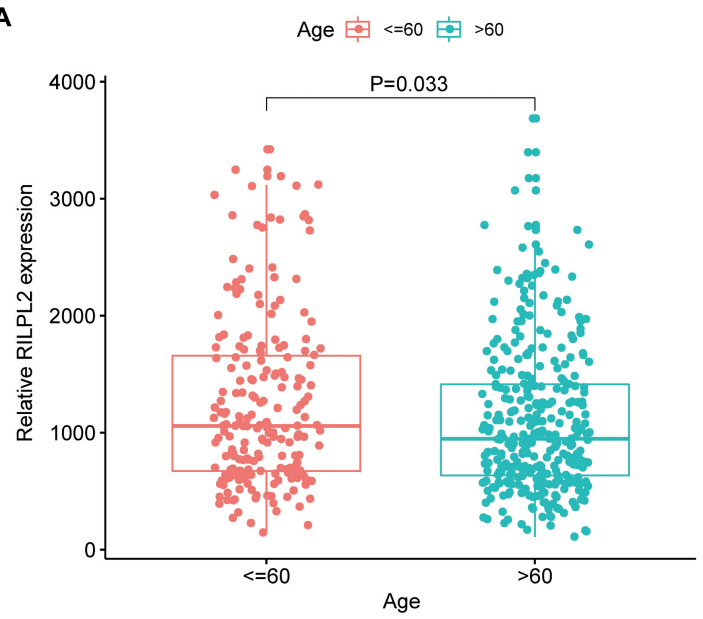

C

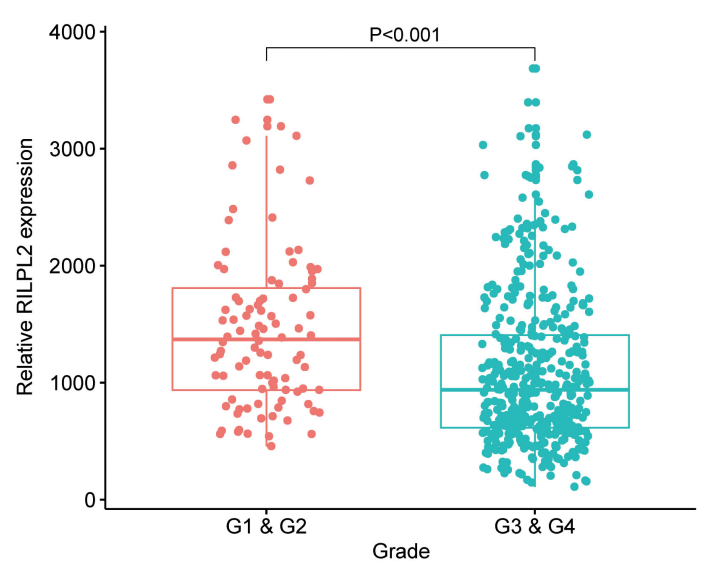

B

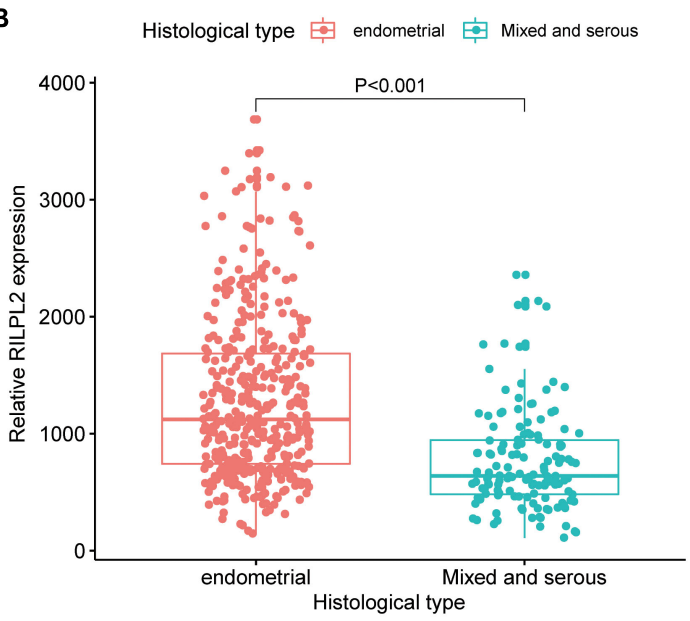

D

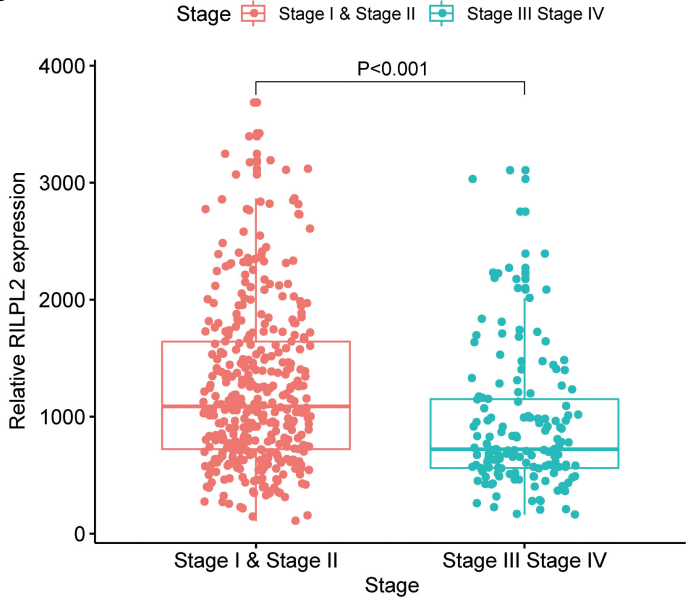

FIGURE 2 | Correlation of RILPL2 expression of and clinicopathologic variables. (A) age, (B) histological type, (C) grade, and (D) stage.

level of RILPL2 by comparing EC tissues with normal tissues. Furthermore, we analyzed the correlation between RILPL2 methylation and its expression level in EC. Besides, the genetic alterations of RILPL2 gene in the TCGA dataset were analyzed by cBioPortal tool (Gao et al., 2013).

\section{WGCNA Analysis}

First, as previously described, differentially expressed genes (DEGs) between EC and normal specimens in the TCGA dataset were screened out using the "limma" package with threshold of adjusted $P$-value $<0.05$ and $|\log 2 \mathrm{FC}|>1$ (Liu et al., 2020b). Next, the expression profile of these DEGs was obtained to establish a gene co-expression network by the weighted gene coexpression network analysis (WGCNA) package in $\mathrm{R}$ language as previously mentioned (Chen et al., 2020). To match the correlations with the nature regulation relationship, we built a scale-free topology by emphasizing the strong correlations and attenuating the weak correlations with the soft threshold power of $\beta=3$ (scale free $R^{2}=0.85$ ). After that, the topological overlap matrix (TOM) was calculated on the basis of adjacency matrices. Finally, we applied the dynamic tree cut algorithm to classify

TABLE 1 | Logistic regression analysis between RILPL2 expression and clinical features.

\begin{tabular}{lccc}
\hline \multicolumn{2}{l}{ Clinical characteristics } & Odds ratio & $\boldsymbol{P}$ value \\
\hline Age & $\leq 60$ vs. $>60$ & $0.99(0.98-1.01)$ & 0.483 \\
Grade & 2 vs. 1 & $0.42(0.23-0.77)$ & 0.005 \\
& 3 vs. 1 & $0.17(0.10-0.28)$ & $<0.001$ \\
& 4 vs. 1 & $0.06(0.01-0.26)$ & $<0.001$ \\
Histology & Endometrial vs. mix & $0.40(0.16-0.95)$ & 0.042 \\
& Endometrial vs. serous & $0.40(0.10-0.28)$ & $<0.001$ \\
Stage & II vs. I & $0.57(0.31-1.03)$ & 0.064 \\
& III vs. I & $0.34(0.22-0.53)$ & $<0.001$ \\
& IV vs. I & $0.22(0.09-0.51)$ & $<0.001$
\end{tabular}


genes according to their expression patterns and merged? gene modules. Gene significance (GS) was deemed as the correlation coefficient between transcriptome expression and module traits. The module eigengene (ME) was calculated as a summary profile for all genes in a module. Module significance (MS) was defined as the correlation coefficient between the module and traits. Module membership (MM) was defined by the correlation coefficient of the module eigengene and transcriptome data. When a gene has $\mathrm{MM}>0.80$, it was supposed to be the modules' representative gene, which might possess potential crucial functions.

\section{Enrichment and PPI Analysis}

Gene Ontology (GO) and Kyoto Encyclopedia of Genes and Genomes (KEGG) enrichment analyses were applied to uncover the potential mechanisms of these genes in the turquoise module using DAVID tool ${ }^{5}$. Besides, the key genes were imported into the STRING database ${ }^{6}$ to construct the protein-protein interaction (PPI) network of gene encoding proteins. We set the minimum interaction score as 0.4 (medium confidence).

\section{RESULTS}

\section{Analysis RILPL2 Expression in EC Patients}

By analyzing the data from TCGA, the expression of RILPL2 in the para-cancerous samples was remarkably higher than that in the tumor specimens $(P<0.001$, Figure 1A). Based on GSE17025 dataset, we observed that RILPL2 also decreased in EC samples compared with para-cancerous samples $(P<0.001$,

${ }^{5}$ https://david-d.ncifcrf.gov/

${ }^{6} \mathrm{https}: / /$ string-db.org/
Figure 1B). In addition, we further validated RILPL2 expression using our recruited cohort, and the result showed that RILPL2 in tumor samples was obviously lower than that of normal control ( $P=0.013$, Figure 1C). Moreover, the level of RILPL2 protein in human endometrium and EC specimens was compared by calculating the data obtained from the HPA database, and the result showed that RILPL2 protein level was lower in EC samples (Figure 1D). To further validate the protein level of RILPL2, the CPTAC analysis in UALCAN was applied. Not surprisingly, the result validated the downregulated RILPL2 in EC samples again $(P<0.001$, Figure 1E).

\section{Association Between RILPL2 Expression and Clinicopathologic Features}

A correlation analysis was performed between RILPL2 expression and corresponding clinical characteristics. As presented in Figure 2, decreased expression of RILPL2 is remarkably related to multiple factors, including age $(P=0.033$, Figure 2A), tumor histological type $(P<0.001$, Figure 2B), grade $(P<0.001$, Figure 2C) and clinical stage $(P<0.001$, Figure 2D). Moreover, as exhibited in Table 1, logistic regression analysis utilizing the median of RILPL2 expression as a classification of the dependent variable indicated that reduced RILPL2 expression was significantly correlated with high grade (Grade 2 vs. Grade $1, P=0.005$; Grade 3 vs. Grade $1, P<0.001$; Grade 4 vs. Grade $1, P<0.001$ ), histological type (Mix vs. Endometrial, $P=0.042$; Serous vs. Endometrial, $P<0.001)$ and pathological stage (Stage III vs. Stage I, $P<0.001$; Stage IV vs. Stage I, $P<0.001)$.

\section{Prognostic Value of RILPL2 in EC Patients and Cox Regression Analysis}

Based on the fact of RILPL2 downregulation in EC, we further focused on its prognostic role in patients with EC. KaplanMeier analysis of the TCGA dataset demonstrated that the

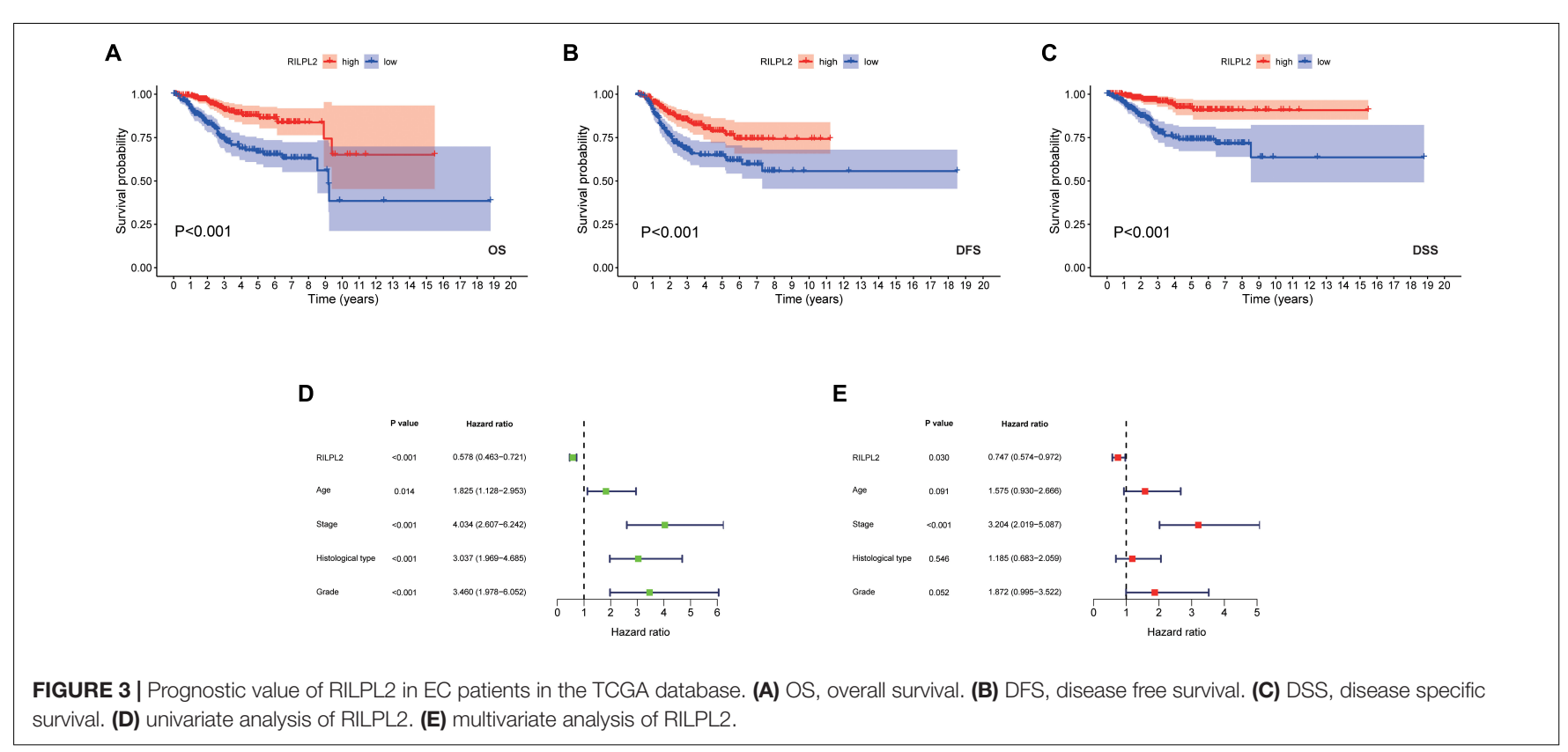


patients with high RILPL2 expression led to significantly better OS $(P<0.001)$ DFS $(P<0.001)$, and DSS $(P<0.001)$ in EC patients compared to the low RILPL2 expression group (Figures 3A-C). Univariate Cox analysis revealed that RILPL2 $(\mathrm{HR}=0.578,95 \% \mathrm{CI}: 0.463-0.721, P<0.001)$ were lowrisk factor, while age, stage, histological type, and grade were high-risk factors (Figure 3D). Furthermore, multivariate Cox analysis showed that RILPL2 (HR $=0.747$, 95\%CI: 0.574$0.972, P=0.030$ ) was independently related to OS (Figure $3 \mathrm{E}$ ), which implied that RILPL2 could be an independent prognostic predictor for EC.

\section{The Relevance of RILPL2 Expression With Immune Infiltration}

Based on the median expression value of RILPL2, 552 EC specimens obtained from the TCGA database were classified into high and low expression cohorts (H-RILPL2 and L-RILPL2 groups). It indicated that the L-RILPL2 group had a higher immune score and stromal score than the H-RILPL2 group via ESTIMATE analysis, while the tumor purity score was inferior (Figure 4A).

The TIMER analysis exhibited that RILPL2 expression had remarkablely positive correlations with $\mathrm{B}$ cells, CD4 $+\mathrm{T}$
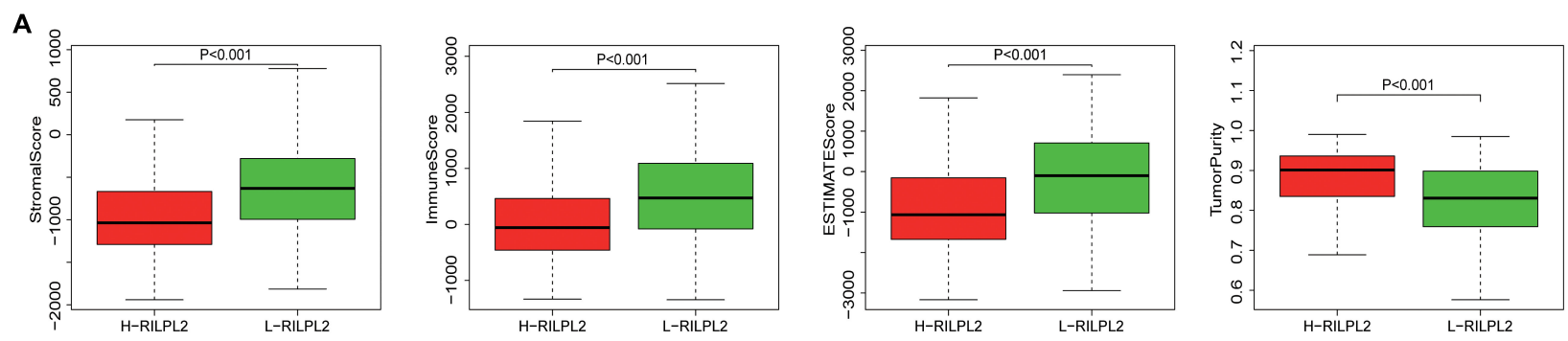

B

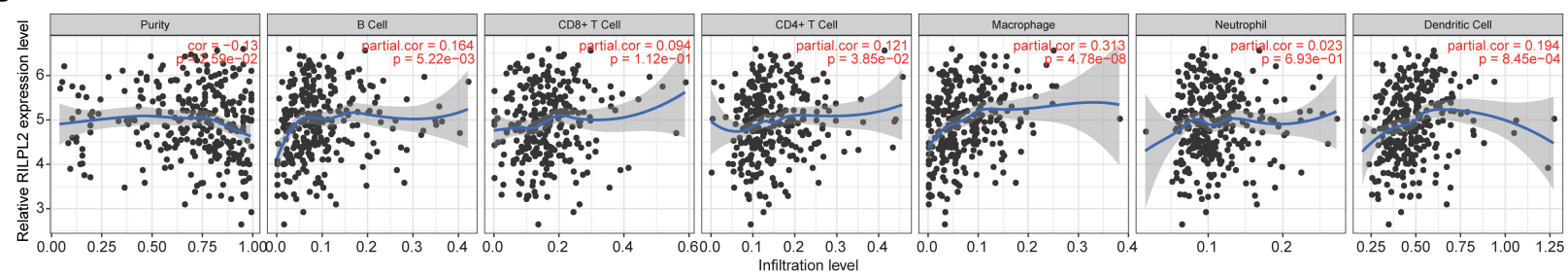

C

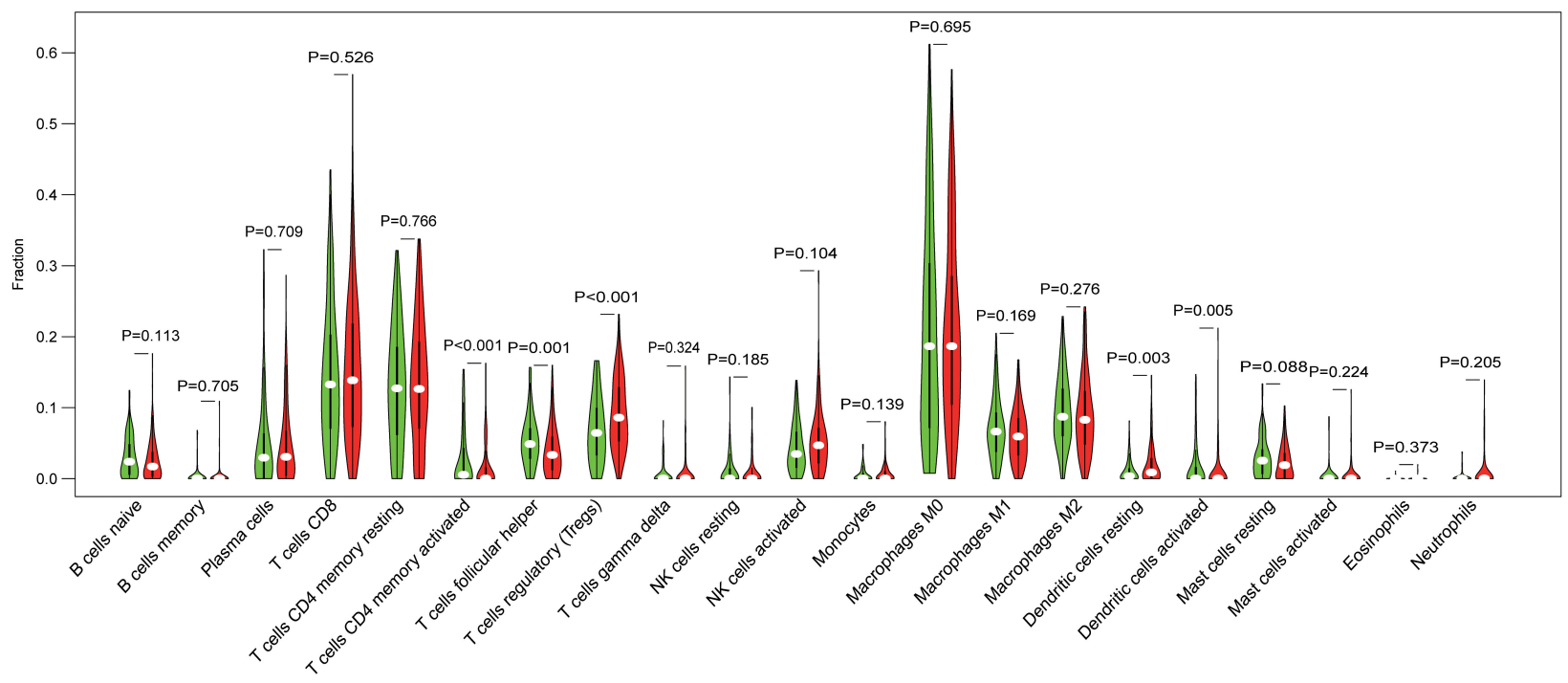

FIGURE 4 | The correlation of TIICs infiltration with RILPL2 expression. (A) Comparison of stromal scores, immune scores, ESTIMATE scores, and tumor purity between the L-RILPL2 and H-RILPL2 groups according to the ESTIMATE tool. (B) TIMER analysis of the correlation between the expression of RILPL2 and six immune cells in EC. (C) The proportion of 22 immune cells in EC tissues in the L-RILPL2 and H-RILPL2 groups. 
cells, macrophages, and DCs in EC (Figure 4B). Moreover, we evaluated the correlations between RILPL2 expression and immune markers of different TIICs subtypes in EC tissues using TIMER database. The analysis exhibited that RILPL2 expression were correlated with the expression of marker genes of CD8 $+\mathrm{T}$ cells, T cells (general), B cells, monocyte, TAMs, M2 macrophage, neutrophils, NK cells, DCs, Th1 cells, Th2 cells, Th17 cells, Treg cells, and T cell exhaustion to varying degrees. Whereas, It revealed unrelated relationship between the expression of RILPL2 and the expression of gene markers for M1 macrophage and Tfh cell in EC samples (Table 2).

To distinguish the variance of the distribution of 22 TIICs between the two groups, CIBERSORT algorithm was applied to analyze EC cases from the TCGA database. The violin plot manifested the ratio differentiation of 22 TICs between EC tumor specimens with L- or H- RILPL2 expression. T cells CD4 memory activated, T cells follicular helper, $\mathrm{T}$ cells regulatory, Dendritic cells resting, and Dendritic cells activated were primary immune cells having a significant relationship with RILPL2 expression (Figure 4C). The proportion of all these five immune cells was increased in H-RILPL2 group compared with L-RILPL2 group.

\section{Methylation and Genetic Alterations of RILPL2 in EC}

The DNA methylation level of RILPL2 was obtained from the TCGA database, and the differentially expressed methylation levels of RILPL2 between EC and para-cancerous specimens were analyzed. The methylation level of RILPL2 in the normal samples was notably lower than that in the tumor samples $(P<0.001$, Figure 5A). Besides, the total methylation level of RILPL2 gene and methylation level of each site all showed significantly negative correlation with its expression $(P<0.001$, Figure 5B and Supplementary Figure 1), which indicated that high methylation level was a significant cause for RILPL2 low expression in EC. Besides, low amplification and mutation rates of RILPL2 were found in EC patients (Figure 5C). Thus, genetic alterations might not be crucial for the dysregulation of RILPL2 in EC.

\section{Analysis of the Potential Mechanisms of RILPL2}

In order to build a weighted co-expression network and identify modules and genes related to RILPL2, 2,573 DEGs between EC and para-cancerous tissues from the TCGA database were submitted to WGCNA. After a series of adjustments for WGCNA parameters, the DEGs were clarified into nine modules by average linkage hierarchical clustering (Supplementary Figures 2A,B, Figures 6A,B). Among these modules, the turquoise module hinted the highest negative correlation with RILPL2 expression (Cor $=-0.45, P<0.001)$ (Figure 6C), which might indicated the RILPL2-involved potential oncogenic mechanisms. Subsequently, forty-two genes in the turquoise module were reserved as key genes (GS > 0.2 and $M M>0.8$ ) (Figure 6D).

To further understand the potential oncogenic mechanisms associated to these hub genes, we next conduct GO and KEGG analysis to perform. "chromosome segregation," "chromosome region," and "DNA-dependent ATPase activity" were the
TABLE 2 | Correlation analysis between RILPL2 and related gene markers of immune cells.

\begin{tabular}{|c|c|c|c|}
\hline Description & Gene markers & $R$ value & $P$ value \\
\hline \multirow[t]{2}{*}{ CD8+ T cell } & CD8A & 0.141 & 0.001 \\
\hline & CD8B & 0.105 & 0.014 \\
\hline \multirow[t]{3}{*}{ T cell (general) } & CD3D & 0.211 & $<0.001$ \\
\hline & CD3E & 0.198 & $<0.001$ \\
\hline & CD2 & 0.193 & $<0.001$ \\
\hline \multirow[t]{2}{*}{ B cell } & CD19 & 0.246 & $<0.001$ \\
\hline & CD79A & 0.157 & $<0.001$ \\
\hline \multirow[t]{2}{*}{ Monocyte } & CD86 & 0.247 & $<0.001$ \\
\hline & CSF1R & 0.319 & $<0.001$ \\
\hline \multirow[t]{3}{*}{ TAM } & CCL2 & 0.135 & 0.002 \\
\hline & CD68 & 0.157 & $<0.001$ \\
\hline & IL10 & -0.111 & 0.010 \\
\hline \multirow[t]{3}{*}{ M1 macrophage } & NOS2 & -0.021 & 0.631 \\
\hline & IRF5 & 0.025 & 0.562 \\
\hline & PTGS2 & 0.072 & 0.092 \\
\hline \multirow[t]{3}{*}{ M2 macrophage } & CD163 & 0.128 & 0.003 \\
\hline & VSIG4 & 0.213 & $<0.001$ \\
\hline & MS4A4A & 0.215 & $<0.001$ \\
\hline \multirow[t]{3}{*}{ Neutrophils } & CEACAM8 & 0.005 & 0.915 \\
\hline & ITGAM & 0.294 & $<0.001$ \\
\hline & CCR7 & 0.182 & $<0.001$ \\
\hline \multirow[t]{7}{*}{ Natural killer cell } & KIR2DL1 & 0.071 & 0.096 \\
\hline & KIR2DL3 & 0.066 & 0.125 \\
\hline & KIR2DL4 & 0.196 & $<0.001$ \\
\hline & KIR3DL1 & 0.022 & 0.615 \\
\hline & KIR3DL2 & 0.035 & 0.419 \\
\hline & KIR3DL3 & 0.079 & 0.066 \\
\hline & KIR2DS4 & 0.028 & 0.517 \\
\hline \multirow[t]{7}{*}{ Dendritic cell } & HLA-DPB1 & 0.325 & $<0.001$ \\
\hline & HLA-DQB1 & 0.218 & $<0.001$ \\
\hline & HLA-DRA & 0.333 & $<0.001$ \\
\hline & HLA-DPA1 & 0.301 & $<0.001$ \\
\hline & CD1C & 0.419 & $<0.001$ \\
\hline & NRP1 & 0.288 & $<0.001$ \\
\hline & ITGAX & 0.262 & $<0.001$ \\
\hline \multirow[t]{5}{*}{ Th1 cell } & TBX21 & 0.155 & $<0.001$ \\
\hline & STAT4 & 0.150 & $<0.001$ \\
\hline & STAT1 & -0.053 & 0.214 \\
\hline & IFNG & 0.026 & 0.540 \\
\hline & TNF & 0.028 & 0.516 \\
\hline \multirow[t]{4}{*}{ Th2 cell } & GATA3 & -0.032 & 0.452 \\
\hline & STAT6 & 0.208 & $<0.001$ \\
\hline & STAT5A & 0.271 & $<0.001$ \\
\hline & IL13 & -0.020 & 0.641 \\
\hline \multirow[t]{2}{*}{ Tfh cell } & BCL6 & 0.024 & 0.582 \\
\hline & IL21 & 0.012 & 0.773 \\
\hline \multirow[t]{2}{*}{ Th17 cell } & STAT3 & 0.200 & $<0.001$ \\
\hline & IL17A & 0.013 & 0.758 \\
\hline \multirow[t]{4}{*}{ Treg cell } & FOXP3 & 0.119 & 0.005 \\
\hline & CCR8 & 0.086 & 0.046 \\
\hline & STAT5B & 0.141 & 0.001 \\
\hline & TGFB1 & 0.032 & 0.453 \\
\hline \multirow[t]{5}{*}{ T cell exhaustion } & PDCD1 & -0.010 & 0.808 \\
\hline & CTLA4 & 0.169 & $<0.001$ \\
\hline & LAG3 & -0.041 & 0.334 \\
\hline & HAVCR2 & 0.244 & $<0.001$ \\
\hline & GZMB & -0.032 & 0.454 \\
\hline
\end{tabular}




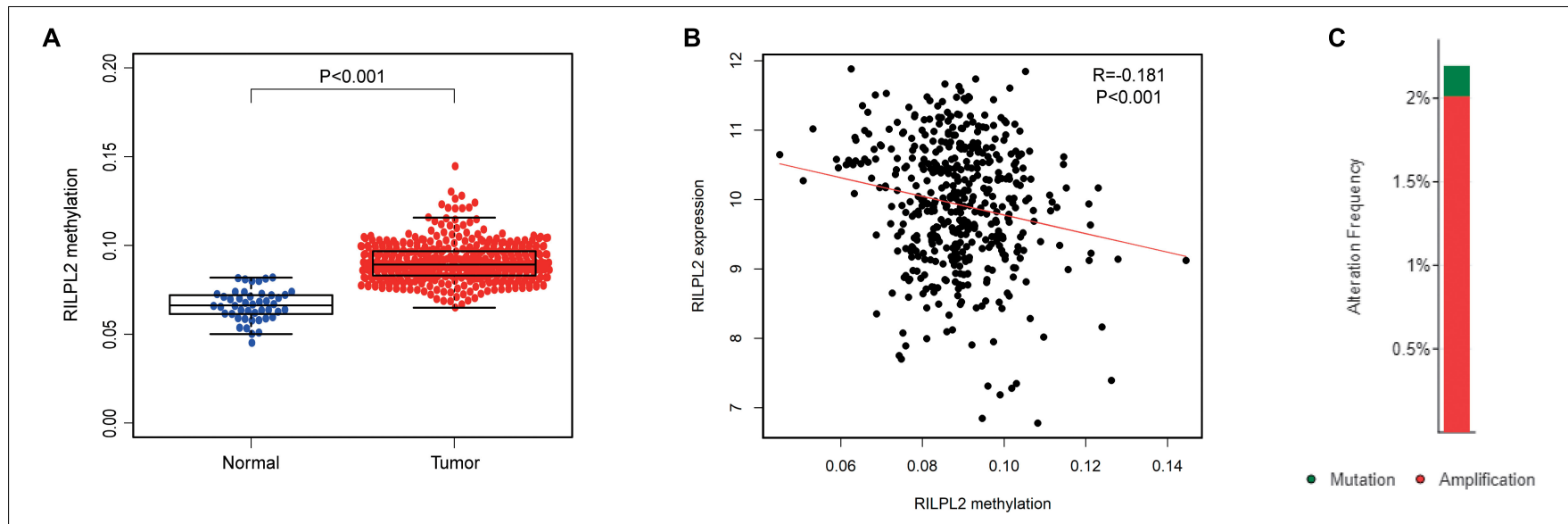

FIGURE 5 | Methylation and mutation analysis of RILPL2 in EC. (A) Methylation level of RILPL2 in EC from the TCGA dataset. (B) Correlation between RILPL2 methylation level and its expression in EC. (C) The variety and proportion of samples with genetic alterations of RILPL2 in EC.

A

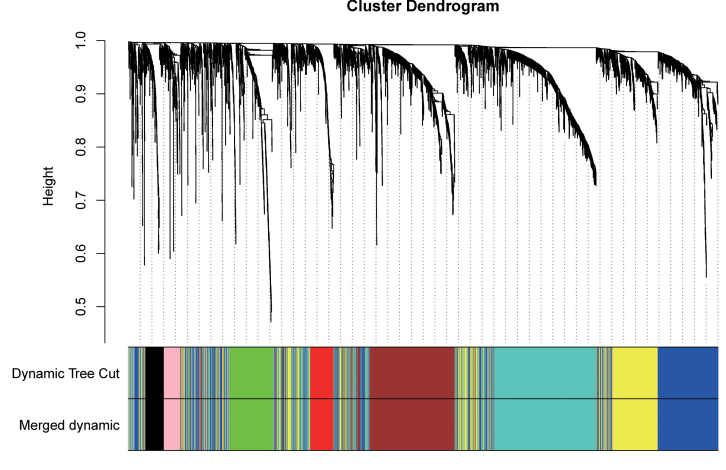

C

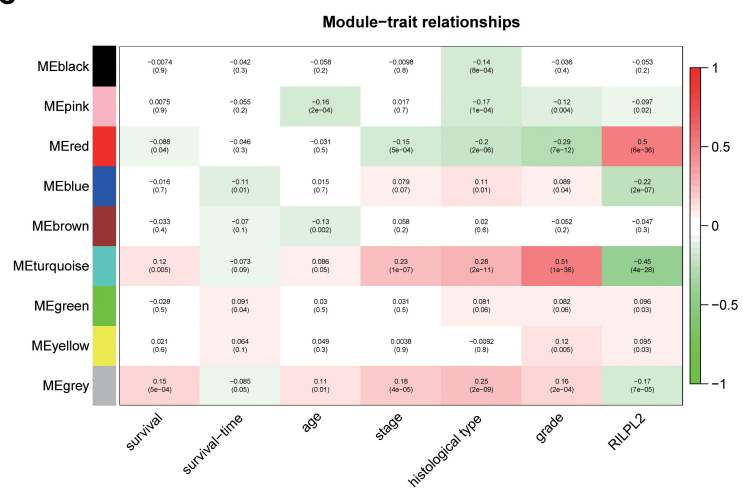

B

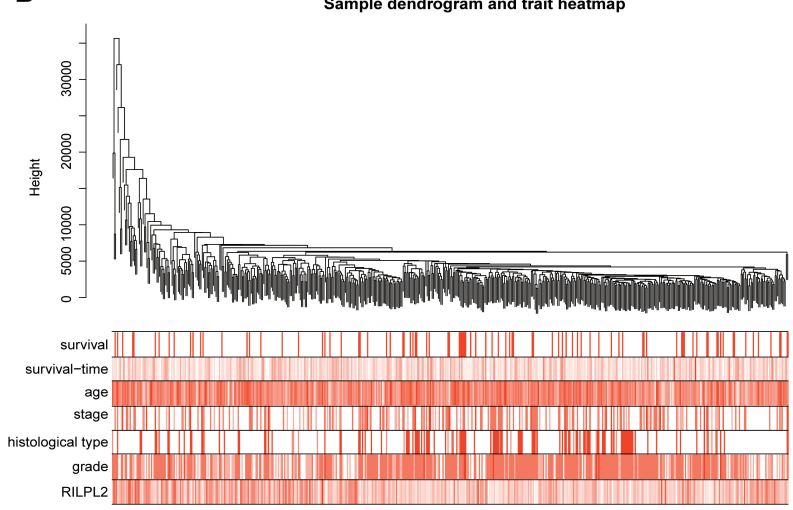

D

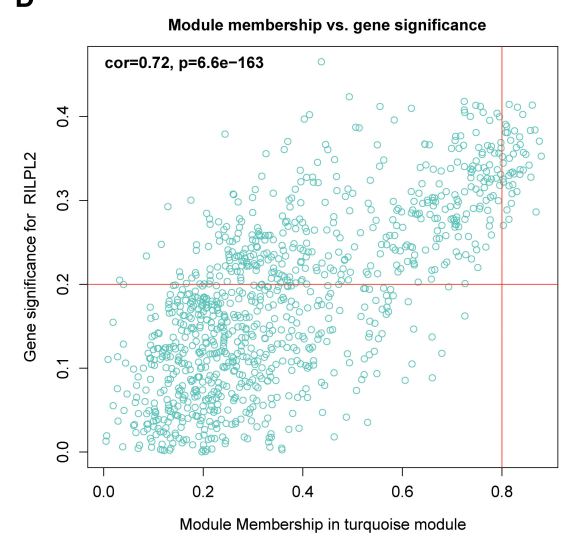

FIGURE 6 | Exploration for genes correlated with RILPL2 in EC. (A) Clustering dendrogram of EC patients from the TCGA dataset. (B) A total of 2,573 DEGs were clustered according to the dissimilarity measure (1-TOM) and were classified into nine modules. (C) A correlation heatmap between module eigengenes and clinical features of EC. (D) Scatter plot of turquoise module eigengenes.

significantly important GO terms for cellular components, biological processes and molecular functions, respectively (Figure 7A). "cell cycle" was the most significant pathway in the
KEGG pathway analysis (Figure 7B). Besides, we established a PPI network with these hub genes and found that a large numbers of cell cycle related genes play key roles (Figure 7C). 

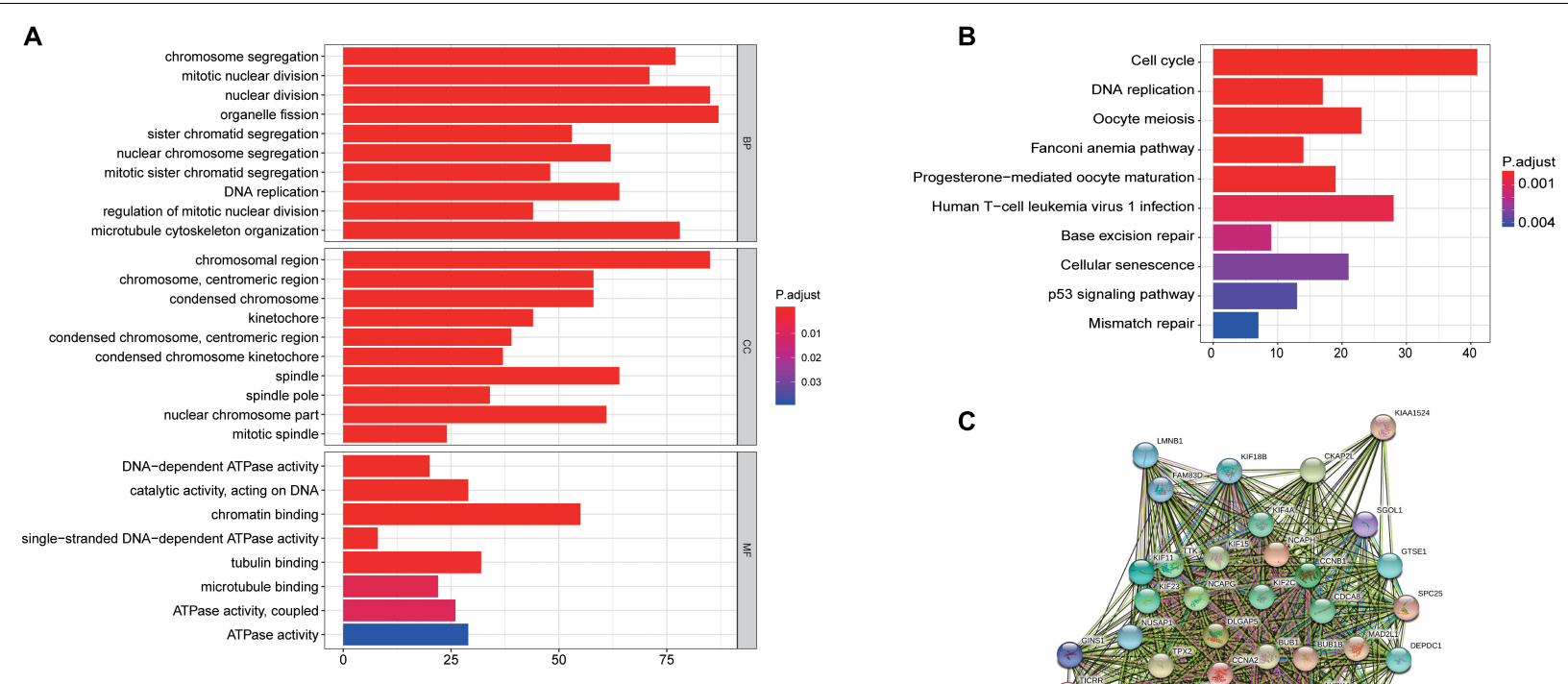

C

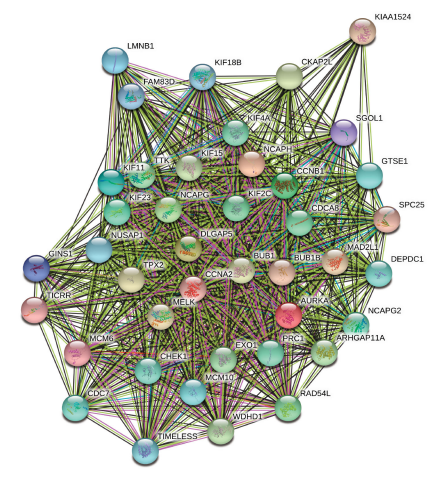

FIGURE 7 | Latent oncogenic mechanisms of RILPL2 in EC. (A) GO analysis of genes in turquoise module eigengenes, top 10 terms were exhibited. (B) KEGG analysis of gene in turquoise module eigengenes, top 10 terms were exhibited. (C) PPIs of hub genes constructed by STRING tool.

\section{DISCUSSION}

Endometrial carcinoma is one of the most widespread malignant tumors in female's genital tract in the world and accounts for approximately 74,000 deaths per year worldwide (Ferlay et al., 2010; Siegel et al., 2017). The incidence of EC is increasing in the range of 10 to 20 per 100,000 women annually, and the age of onset is younger than in prior years (Moore and Brewer, 2017). The combination of surgery, radiotherapy, and chemotherapy is the current gold standards for treatment of EC patients. However, the 5-year survival rate is approximately $45-60 \%$ and $15-25 \%$ for stage III and stage IV EC, respectively, which led to the majority of EC-related deaths (Amant et al., 2005). Hence, it is of critical importance to identify credible biomarkers for early diagnosis, early initiation of treatments and improved prognosis of EC.

In this work, sequential data filtering was performed from the TCGA database, which determined the identification of the key gene RILPL2. The relationship between RILPL2 expression and clinical factors was analyzed by logistic regression analysis and clinical relevance filtering. Besides, we used Kaplan-Meier analysis, as well as univariate and multivariate Cox analysis to assess the associations between RILPL2 expression and survival outcomes. Not only that, we analyzed the relevance of RILPL2 expression with immune cells infiltration. Finally, the WGCNA analysis was conducted to perform the underlying mechanisms of RILPL2.

Previous research indicated that RILPL2 widely expressed in most tissues, including brain, heart, lung, liver, kidney, pancreas, and placenta (Wang et al., 2004). To our best knowledge, no available studies have revealed the expression of
RILPL2 and its underlying prognostic impact in EC. However, relative studies indicated that RILPL2 showed a significant correlation with breast cancer by conducting series of literatures of RILPL2. Chen et al. revealed that RILPL2 expression in breast cancer tissues is lower than that in para-cancerous tissues, and that RILPL2 upregulation is associated with prolonged prognosis. Overexpression of RILPL2 inhibited breast cancer cell proliferation and metastasis in vitro and in vivo. Besides, the interaction of exogenous RILPL2 with TUBB3 resulted in the downregulation of breast cancer cell proliferation and migration and upregulation of PTEN expression by promoting destabilization of TUBB3 (Chen et al., 2019).

The present study focused on the potential role of RILPL2 in EC. We identified that RILPL2 was expressed differently in normal tissues and EC tissues. Kaplan-Meier analysis exhibited that high level of RILPL2 was notably related to better prognosis in patients with EC. From the result of univariate and multivariate Cox analysis, we found that RILPL2 was a highrisk factor and could be served as an independent indicator to forecast the clinical outcome of patients with EC. Besides, The ESTIMATE, TIMER, and CIBERSORT analysis both suggested RILPL2 might play a critical role in regulating immune cell infiltration in EC and is related to immune response. The fraction of immune cell infiltration plays crucial roles in tumor growth, metastasis, and therapeutic resistance (Waniczek et al., 2017). For example, CD8 + cytotoxic $\mathrm{T}$ cell serves as crucial role to destroy tumor cells in many cancers (Joyce and Fearon, 2015). Macrophages are classified into two classifications with various specific functions: M1, with a proinflammatory function, and M2, with an anti-inflammatory or wound healing effect 
(Demagny et al., 2014; Murray et al., 2014). Recent years, bioinformatics-dependent identification of immune-related biomarkers has been emerging, and increasing numbers of genes are found to be associated with tumor immunity (He et al., 2020; Mei et al., 2020). Our current research suggested that the expression of RILPL2 may regulate immune infiltration of multiple TIICs in the immune microenvironment of EC specimens, thus directly and/or indirectly regulating immune monitor and influencing progression of tumor.

\section{CONCLUSION}

In summary, accumulating evidence on the tumorigenesis and development of RILPL2 in EC were acknowledged and preliminarily presents its potential as a promising diagnostic and prognostic indicator. Our results suggest that RILPL2 is decreased in EC and low RILPL2 expression is related to grade of malignancy and poor prognosis. In addition, our research also indicates that RILPL2 is closely correlated with immune infiltration in EC. Overall, these findings provide a theoretical basis for next underlying studies to validate the function of RILPL2 in EC.

\section{DATA AVAILABILITY STATEMENT}

The datasets presented in this study can be found in online repositories. The names of the repository/repositories

\section{REFERENCES}

Amant, F., Moerman, P., Neven, P., Timmerman, D., Van Limbergen, E., and Vergote, I. (2005). Endometrial cancer. Lancet 366, 491-505.

Bokhman, J. V. (1983). Two pathogenetic types of endometrial carcinoma. Gyneco.l Oncol. 15, 10-17. doi: 10.1016/0090-8258(83)90111-7

Chandrashekar, D. S., Bashel, B., Balasubramanya, S. A. H., Creighton, C. J., PonceRodriguez, I., Chakravarthi, B., et al. (2017). UALCAN: a portal for facilitating tumor subgroup gene expression and survival analyses. Neoplasia 19, 649-658. doi: 10.1016/j.neo.2017.05.002

Chen, G., Sun, L., Han, J., Shi, S., Dai, Y., and Liu, W. (2019). RILPL2 regulates breast cancer proliferation, metastasis, and chemoresistance via the TUBB3/PTEN pathway. Am. J. Cancer Res. 9, 1583-1606.

Chen, J., Cai, Y., Xu, R., Pan, J., Zhou, J., and Mei, J. (2020). Identification of four hub genes as promising biomarkers to evaluate the prognosis of ovarian cancer in silico. Cancer Cell Int. 20:270.

Creasman, W. T., Kohler, M. F., Odicino, F., Maisonneuve, P., and Boyle, P. (2004). Prognosis of papillary serous, clear cell, and grade 3 stage I carcinoma of the endometrium. Gynecol. Oncol. 95, 593-596. doi: 10.1016/j.ygyno.2004.08.019

Day, R. S., McDade, K. K., Chandran, U. R., Lisovich, A., Conrads, T. P., Hood, B. L., et al. (2011). Identifier mapping performance for integrating transcriptomics and proteomics experimental results. BMC Bioinform. 12:213. doi: 10.1186/1471-2105-12-213

Demagny, H., Araki, T., and De Robertis, E. M. (2014). The tumor suppressor Smad4/DPC4 is regulated by phosphorylations that integrate FGF, Wnt, and TGF-beta signaling. Cell Rep. 9, 688-700. doi: 10.1016/j.celrep.2014.09.020

Dolan, P. T., Zhang, C., Khadka, S., Arumugaswami, V., Vangeloff, A. D., Heaton, N. S., et al. (2013). Identification and comparative analysis of hepatitis C virus-host cell protein interactions. Mol. Biosyst. 9, 3199-3209. doi: 10.1039/ c3mb70343f and accession number(s) can be found in the article/ Supplementary Material.

\section{ETHICS STATEMENT}

The studies involving human participants were reviewed and approved by Nantong Third People's Hospital Affiliated to Nantong University in 2020. The patients/participants provided their written informed consent to participate in this study. The animal study was reviewed and approved by Nantong Third People's Hospital Affiliated to Nantong University in 2020.

\section{AUTHOR CONTRIBUTIONS}

HZ conceived the study and participated in the study design, performance, and manuscript writing. JHL, MX, ZW, and JQL conducted the bioinformatics analysis. HZ, YY, and SY revised the manuscript. All authors read and approved the final manuscript.

\section{SUPPLEMENTARY MATERIAL}

The Supplementary Material for this article can be found online at: https://www.frontiersin.org/articles/10.3389/fmolb. 2021.670893/full\#supplementary-material

Ferlay, J., Shin, H. R., Bray, F., Forman, D., Mathers, C., and Parkin, D. M. (2010) Estimates of worldwide burden of cancer in 2008: GLOBOCAN 2008. Int. J. Cancer 127, 2893-2917. doi: 10.1002/ijc.25516

Fukuda, M., Kanno, E., Ishibashi, K., and Itoh, T. (2008). Large scale screening for novel rab effectors reveals unexpected broad Rab binding specificity. Mol. Cell Proteomics 7, 1031-1042. doi: 10.1074/mcp.m700569-mcp200

Gao, J., Aksoy, B. A., Dogrusoz, U., Dresdner, G., Gross, B., Sumer, S. O., et al. (2013). Integrative analysis of complex cancer genomics and clinical profiles using the cBioPortal. Sci Signal. 6, 11.

He, D., Zhang, X., and Tu, J. (2020). Diagnostic significance and carcinogenic mechanism of pan-cancer gene POU5F1 in liver hepatocellular carcinoma. Cancer Med. 9, 8782-8800. doi: 10.1002/cam4.3486

Johansson, M., Rocha, N., Zwart, W., Jordens, I., Janssen, L., Kuijl, C., et al. (2007). Activation of endosomal dynein motors by stepwise assembly of Rab7-RILPp150Glued, ORP1L, and the receptor betall spectrin. J. Cell Biol. 176, 459-471. doi: $10.1083 /$ jcb. 200606077

Joyce, J. A., and Fearon, D. T. (2015). T cell exclusion, immune privilege, and the tumor microenvironment. Science 348, 74-80. doi: 10.1126/science.aaa6204

Li, T., Fan, J., Wang, B., Traugh, N., Chen, Q., Liu, J. S., et al. (2017). TIMER: a web server for comprehensive analysis of tumor-infiltrating immune cells. Cancer Res. 77, e108-e110.

Liu, J., Lichtenberg, T., Hoadley, K. A., Poisson, L. M., Lazar, A. J., Cherniack, A. D., et al. (2018). An integrated TCGA pan-cancer clinical data resource to drive high-quality survival outcome analytics. Cell 173, 400-416.e11. *400-416.e11

Liu, J., Mei, J., Li, S., Wu, Z., and Zhang, Y. (2020a). Establishment of a novel cell cycle-related prognostic signature predicting prognosis in patients with endometrial cancer. Cancer Cell Int. 20:329.

Liu, J., Wu, Z., Sun, R., Nie, S., Meng, H., Zhong, Y., et al. (2020b). Using mRNAsi to identify prognostic-related genes in endometrial carcinoma based on WGCNA. Life Sci. 258:118231. doi: 10.1016/j.lfs.2020.118231

Matsui, T., Ohbayashi, N., and Fukuda, M. (2012). The rab interacting lysosomal protein (RILP) homology domain functions as a novel effector domain for 
small GTPase Rab36: Rab36 regulates retrograde melanosome transport in melanocytes. J. Biol. Chem. 287, 28619-28631. doi: 10.1074/jbc.m112.370544

Mei, J., Wang, R., Xia, D., Yang, X., Zhou, W., Wang, H., et al. (2020). BRCA1 Is a Novel prognostic indicator and associates with immune cell infiltration in hepatocellular Carcinoma. DNA Cell Biol. 39, 1838-1849. doi: 10.1089/dna. 2020.5644

Moore, K., and Brewer, M. A. (2017). Endometrial cancer: is this a new disease? Am. Soc. Clin. Oncol. Educ. Book 37, 435-442. doi: 10.14694/edbk_175666

Murray, P. J., Allen, J. E., Biswas, S. K., Fisher, E. A., Gilroy, D. W., Goerdt, S., et al. (2014). Macrophage activation and polarization: nomenclature and experimental guidelines. Immunity. 41, 14-20. doi: 10.1016/j.immuni.2014.06. 008

Newman, A. M., Liu, C. L., Green, M. R., Gentles, A. J., Feng, W., Xu, Y., et al. (2015). Robust enumeration of cell subsets from tissue expression profiles. Nat. Methods 12, 453-457. doi: 10.1038/nmeth.3337

Siegel, R. L., Miller, K. D., and Jemal, A. (2017). Cancer statistics, 2017. CA Cancer J. Clin. 67, 7-30. doi: 10.3322/caac.21387

Siegel, R. L., Miller, K. D., and Jemal, A. (2019). Cancer statistics, 2019. CA Cancer J. Clin. 69, 7-34.

Sorosky, J. I. (2012). Endometrial cancer. Obstet. Gynecol. 120(2 Pt. 1), 383-397.

Uhlen, M., Fagerberg, L., Hallstrom, B. M., Lindskog, C., Oksvold, P., Mardinoglu, A., et al. (2015). Proteomics. Tissue-based map of the human proteome. Science $347: 1260419$.
Wang, T., Wong, K. K., and Hong, W. (2004). A unique region of RILP distinguishes it from its related proteins in its regulation of lysosomal morphology and interaction with Rab7 and Rab34. Mol. Biol. Cell 15, 815-826. doi: 10.1091/mbc.e03-06-0413

Waniczek, D., Lorenc, Z., Snietura, M., Wesecki, M., Kopec, A., and MucWierzgon, M. (2017). Tumor-associated macrophages and regulatory T cells infiltration and the clinical outcome in colorectal cancer. Arch. Immunol. Ther. Exp. (Warsz). 65, 445-454. doi: 10.1007/s00005-017-0463-9

Yoshihara, K., Shahmoradgoli, M., Martinez, E., Vegesna, R., Kim, H., Torres-Garcia, W., et al. (2013). Inferring tumour purity and stromal and immune cell admixture from expression data. Nat. Commun. $4: 2612$.

Conflict of Interest: The authors declare that the research was conducted in the absence of any commercial or financial relationships that could be construed as a potential conflict of interest.

Copyright (c) $2021 \mathrm{Liu}, \mathrm{Xu}, \mathrm{Wu}$, Yang, Yuan, Liang and Zhu. This is an open-access article distributed under the terms of the Creative Commons Attribution License (CC BY). The use, distribution or reproduction in other forums is permitted, provided the original author(s) and the copyright owner(s) are credited and that the original publication in this journal is cited, in accordance with accepted academic practice. No use, distribution or reproduction is permitted which does not comply with these terms. 